\title{
Medaka: a promising model animal for comparative population genomics
}

\author{
Yoshifumi Matsumoto ${ }^{\dagger 1,7}$, Hiroki Oota*†1, Yoichi Asaoka ${ }^{2}$, Hiroshi Nishina ${ }^{2}$, \\ Koji Watanabe ${ }^{3}$, Janusz M Bujnicki ${ }^{4}, 5,6$, Shoji Oda ${ }^{1}$, Shoji Kawamura ${ }^{1}$ and \\ Hiroshi Mitani*1
}

\begin{abstract}
Address: ${ }^{1}$ Department of Integrated Biosciences, Graduate School of Frontier Sciences, University of Tokyo, Tokyo, Japan, ${ }^{2}$ Department of Developmental and Regenerative Biology, Medical Research Institute, Tokyo Medical and Dental University, Tokyo, Japan, ${ }^{3}$ FUJIYA CO., LTD., Kanagawa, Japan, ${ }^{4}$ Department of Medical Genome Sciences, Graduate School of Frontier Sciences, University of Tokyo, Tokyo, Japan, ${ }^{5}$ International Institute of Molecular and Cell Biology, Warsaw, Poland, 6 Institute of Molecular Biology and Biotechnology, Faculty of Biology, Adam Mickiewicz University, Poznan, Poland and ${ }^{7}$ Laboratory for Behavioral and Developmental Disorders, Brain Science Institute, RIKEN, Saitama, Japan

Email: Yoshifumi Matsumoto - miraihe08@brain.riken.jp; Hiroki Oota* - hiroki_oota@k.u-tokyo.ac.jp; Yoichi Asaoka - yasaoka.dbio@mri.tmd.ac.jp; Hiroshi Nishina - nishina.dbio@mri.tmd.ac.jp; Koji Watanabe - peko-poko-fujiya@ab.inbox.ne.jp; Janusz M Bujnicki - iamb@genesilico.pl; Shoji Oda - odasho@k.u-tokyo.ac.jp; Shoji Kawamura - kawamura@k.u-tokyo.ac.jp; Hiroshi Mitani* - mitani@k.u-tokyo.ac.jp

* Corresponding authors †Equal contributors
\end{abstract}

Published: 10 May 2009

BMC Research Notes 2009, 2:88 doi:10.1 186/1756-0500-2-88

This article is available from: http://www.biomedcentral.com//756-0500/2/88

(C) 2009 Oota et al; licensee BioMed Central Ltd.

This is an Open Access article distributed under the terms of the Creative Commons Attribution License (http://creativecommons.org/licenses/by/2.0), which permits unrestricted use, distribution, and reproduction in any medium, provided the original work is properly cited.
Received: 25 March 2009
Accepted: 10 May 2009

\begin{abstract}
Background: Within-species genome diversity has been best studied in humans. The international HapMap project has revealed a tremendous amount of single-nucleotide polymorphisms (SNPs) among humans, many of which show signals of positive selection during human evolution. In most of the cases, however, functional differences between the alleles remain experimentally unverified due to the inherent difficulty of human genetic studies. It would therefore be highly useful to have a vertebrate model with the following characteristics: (I) high within-species genetic diversity, (2) a variety of gene-manipulation protocols already developed, and (3) a completely sequenced genome. Medaka (Oryzias latipes) and its congeneric species, tiny fresh-water teleosts distributed broadly in East and Southeast Asia, meet these criteria.
\end{abstract}

Findings: Using Oryzias species from 27 local populations, we conducted a simple screening of nonsynonymous SNPs for II genes with apparent orthology between medaka and humans. We found medaka SNPs for which the same sites in human orthologs are known to be highly differentiated among the HapMap populations. Importantly, some of these SNPs show signals of positive selection.

Conclusion: These results indicate that medaka is a promising model system for comparative population genomics exploring the functional and adaptive significance of allelic differentiations. 


\section{Background}

The accumulation of human genetic polymorphism data provided by sources such as the international HapMap project $[1,2]$ has revealed a number of SNP sites with markedly different allele frequencies among human populations. Such data make systematic searches for diseasecausing or drug-responsive genomic regions possible $[3,4]$, and the accumulated SNP data can also provide compelling evidence of positive selection during human evolution $[5,6]$. An inevitable issue, however, is that mutagenesis and/or crossing-over experiments to elucidate functional differences between alleles at these polymorphic sites are practically impossible in humans. A vertebrate model animal with a broad geographic distribution and documented high genetic polymorphism could serve as a "natural library" of genetic variation in humans for orthologous genes that could be under similar selective pressures.

The medaka (Oryzias latipes) is a notable candidate for such a model animal. This small freshwater fish is found in East Asia with closely related congeneric species broadly distributed throughout Southeast Asia, and it has a long history of use as an experimental animal since the early 20th century. A number of inbred medaka strains have been established, and transgenesis and mutagenesis protocols have been developed, suggesting that medaka has great potential for use in systematic genetic analyses [7-10]. Medaka genome sequences are also available [11]. The greatest advantage of using medaka is its enormous genetic diversity compared to the other fish models (zebrafish, pufferfish, etc.), with the average nucleotide difference of $3.4 \%$ between two inbred medaka strains being the highest among any vertebrates thus far documented [11]. In this study, our purpose is to assess the validity of medaka as a useful resource of comparative population genomics.

\section{Methods}

\section{Medaka strains}

Japanese medaka (Oryzias latipes) populations consist of four geographical populations. We selected 24 wild-type strains from the Japanese medaka (see Additional file 1) and three closely related congeneric species (O. curvinotus, O. luzonensis and O. celebensis; see Additional file 2). We also examined an inbred strain (Hd-rR) of Southern Japanese origin.

\section{PCR-direct sequence, mRNA extraction and CDNA sequence}

We selected 11 genes for the screening of madaka SNPs (Table 1). The flow chart of the target gene selection is shown in Figure 1a. The PCR primers were designed on the basis of the medaka genomic sequences [11] corresponding to those of humans where high- $F_{\mathrm{st}}$ SNPs are found (Fig. 1b). The PCR performed using genomic DNA extracted from medaka fins or bodys as template. To isolate entire oRTTN sequences, mRNAs were prepared from the embryos of seven strains, because we had already confirmed by in situ hybridization that oRTTN was expressed in early developmental stages. oRTTN genes were PCR amplified with primer pairs designed using the oRTTN sequence predicted from the medaka genome project (Hd-rR strain)[11]. The PCR products were directly sequenced using an ABI PRISM 3130-Avant Genetic Analyzer (Applied Biosystems Japan, Tokyo, Japan) and a total of $\sim 340 \mathrm{~kb}$ of DNA sequences were obtained. The primer sequences and the determined sequences have been deposited in the international GenBank/DDBJ/ EMBL nucleotide sequence database [accession nos. AB435679 - AB435956]. The thermocycling conditions are available on request.

\section{Statistical and phylogenetic analysis}

Nucleotide sequences were aligned using CLUSTALW [12]. The pairwise $d N$ and $d S$ values among strains of 11 genes were calculated by DnaSP Software (version 4.0) according to the Nei-Gojobori method [13]. Insertions and deletions (indels) were excluded from analysis. For the entire nucleotide sequence of RTTN, the $d \mathrm{~N}$ - $d \mathrm{~S}$ and $p$ values were calculated by MEGA 4 [14] according to the Nei-Gojobori method with statistical significance tested by Z-tests.

\section{Protein structure prediction}

The GeneSilico metaserver [15] was used to predict protein secondary structure and order/disorder, and to carry out fold-recognition (i.e. match the query sequence with structurally characterized templates). Potential phosphorylation sites were predicted using a semi-independent component of the metaserver available at the URL http:// genesilico.pl/Phosphoserver/. For the THEA2 protein, the metaserver indicated very high similarity (PCONS score 3.28) of residues 1-360 (human numbering) to known Acyl-CoA hydrolase structures (e.g. 2gvh in the Protein Data Bank) and high similarity of residues 360-607 (PCONS score 2.00) to lipid transfer proteins from the STAR family (e.g. $1 \ln 1$ in the PDB). Long regions of intrinsic conformational disorder were predicted for loops connecting structural domains (around residues 160-200 and 340-370). For the RTTN protein, the metaserver identified the $\alpha$-helical armadillo domain of $\beta$-catenin $(\underline{1 i 7 w}$ in Protein Data Bank) as the best modeling template, in particular for residues 1-120, with a high confidence score (PCONS score 1.67). Long regions of structural disorder, devoid of secondary and tertiary structure, were predicted for residues $120-160$ and $280-370$. Threedimensional structural models of the ordered (i.e. stably folded) parts of THEA2 and RTTN proteins were generated and optimized using the FRankenstein's Monster method 
a

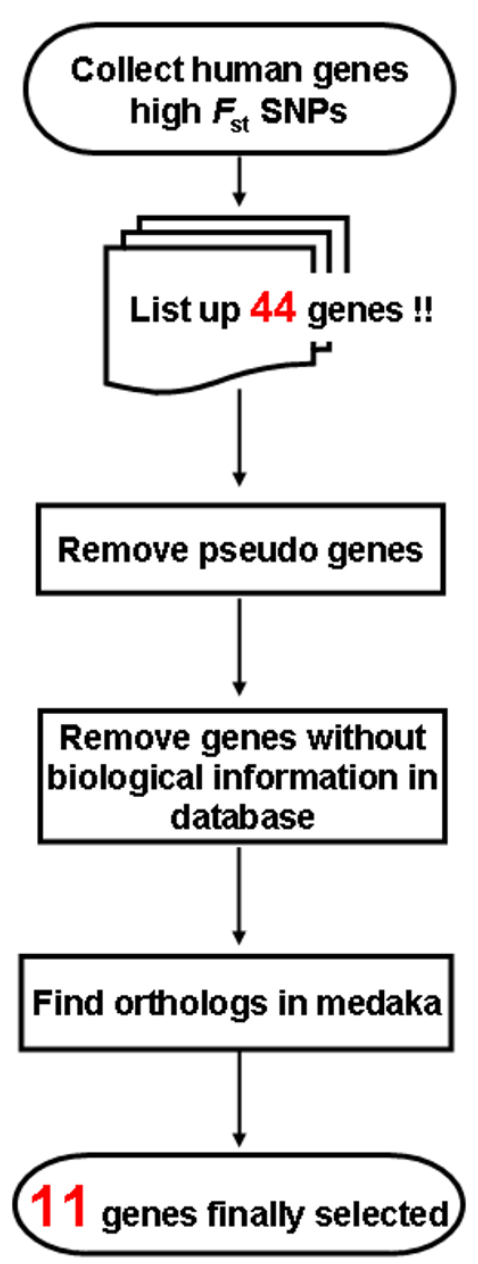

b

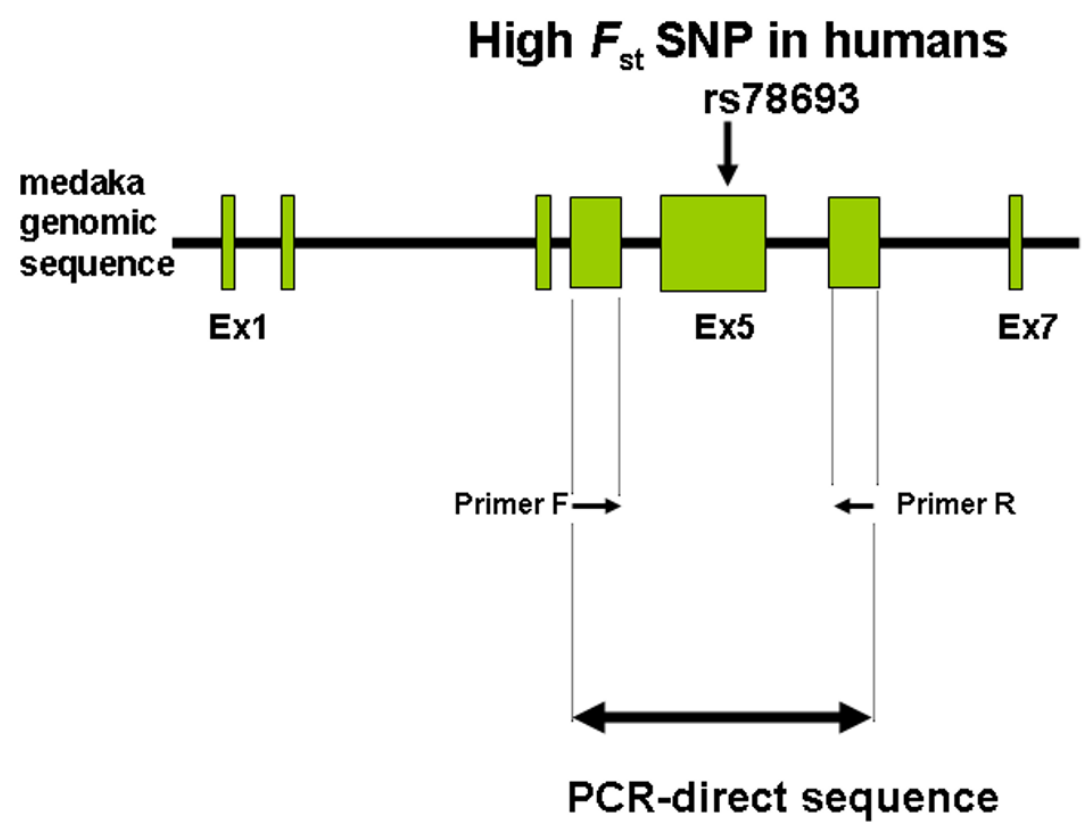

Figure I

(a) Flow chart of targeted gene selection, and (b) a schema of the SNP screening method. We first focused on 44 genes with allele frequencies highly differentiated among human populations, including the 27 genes listed in Table nine of the first HapMap paper [I] based on high $F_{\text {st }}$ values for nonsynonymous SNPs and the 17 genes listed in Table S4 of Sabeti et al. (2006) in the category "population differentiation." A SNP site with a $F_{\text {st }}$ value higher than the genome average represents higher population differentiation at this site [26], possibly driven by natural selection [27-30]. Secondly, from the 44 genes, we removed pseudogenes, genes with unclear annotation and genes without biological information in the database. Thirdly, we chose genes for which only a single gene was assigned as an ortholog in the medaka genome by searching the Ensembl database http://www.ensembl.org/index.html. After applying these selection criteria, II genes were subjected to the SNP screening (Table I).

[16]. The final models were evaluated as good quality by the PROQ server [17]. The models were expected to exhibit a root mean square deviation to the true structures in the order of 2-4 $\AA$, suggesting that they are sufficiently reliable to make functional predictions at the level of individual amino acid residues. The atomic details of these models, however, must be taken with a grain of salt.

\section{Results and discussion}

Of the 11 genes, we found that medaka THEA2 (BFIT2) contained a nonsynonymous SNP at the exactly same site where a high $F_{\mathrm{st}}$ is observed in humans (rs1702003 in exon 6: see the HapMap database; Fig. 2). THEA2 is known to be a temperature responsive gene, and it is expressed in brown adipose tissue (BAT) in response to cold stress in mice [18]. The genotype frequencies at rs 1702003 are $98.3 \%$ G/G and 1.7\% G/A in Europeans and $100 \% \mathrm{~A} / \mathrm{A}$ in East Asians and Africans. This could suggest that the European-specific allele of the cold-inducible gene is an adaptation of Europeans to the cold environment around 40,000 years ago when early modern humans expanded to Europe. Interestingly, only Philip- 
Table I: The II genes examined in this study

\begin{tabular}{ll}
\hline Gene & Gene ontology "biological process" annotation \\
\hline ALDH2 & alcohol metabolic process \\
EDAR & I-kappaB kinase/NF-kappaB cascade \\
F2 & coagulation factor II \\
GRK4 & regulation of G-protein coupled receptor protein signaling pathway \\
LCT & Lactase \\
RTTN & required for axial rotation and left-right specification \\
SLC24A5 & solute carrier family 24, member 5 \\
SLC30A9 & solute carrier family 30 (zinc transporter), member 9 \\
SLC45A2 & solute carrier family 45, member 2 \\
LWS & opsin I (cone pigments), long-wave-sensitive (color blindness, protan) \\
THEA2 & response to temperature stimulus \\
\hline
\end{tabular}

Hd-rR

North Japanese

East Korea

O. luzonensis

O. celebensis

O. curvinotus

Human

Hd-rR

North Japanese

East Korea

o. luzonensis

O. celebensis

O. curvinotus

Human

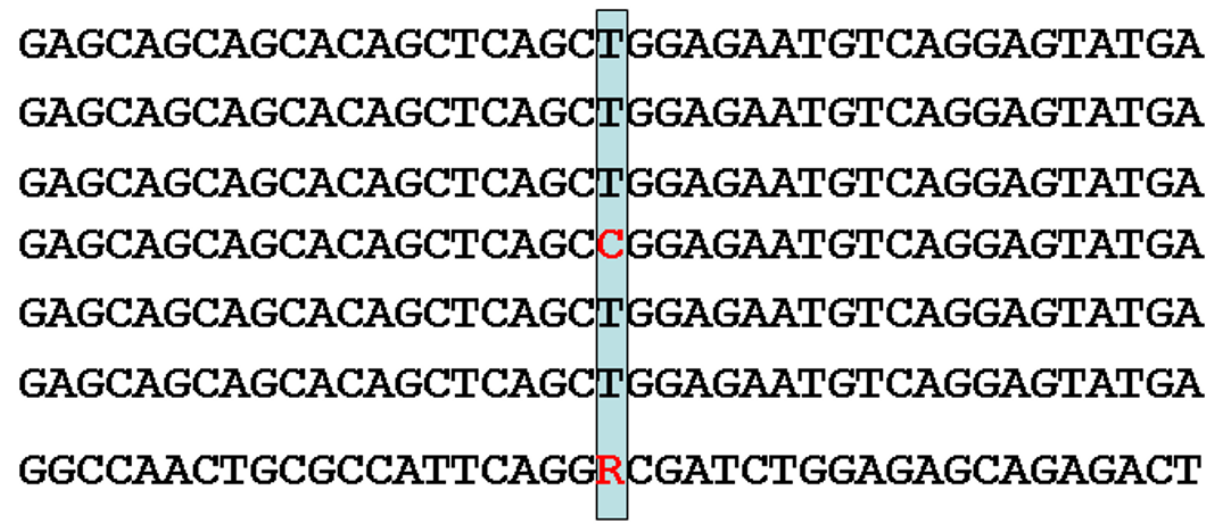

RMRLIHAEIMTDLLSSSTAQL GECQEYEGAVPAERTRVESV RMRLIHAEIMTDLLSSSTAQI GECQEYEGAVPAERTRVESV RMRLIHAEIMTDLLSSSTAQL GECQEYEGAVPAERTRVESV RMRLIHAEIITDLLSSSTAQP GECQEYEGAVPAERTRVESV RMRLIHAEIITDLLSSSTAQIGECQEYEGAVPAERTRVESV RMRLIHAEIITDLLSSSTAQL GECQEYEGAVPAERTRVESV RMRLVYADTIKDLLANCAIQXDLESRDCSRMVPAEKTRVES $\mathrm{D} / \mathrm{G}$

Figure 2

Nucleotide (upper) and amino acid (lower) sequence alignments of THEA2. Hd-rR is the inbred strain derived from the southern Japanese population for which the complete genome sequence has been determined [II]. All three (Hd-rR, Northern Japanese and East Korea) are Oryzias latipes. The others are closely related species. 
pine medaka (Oryzias luzonensis), inhabiting a warmer environment, has a different allele from the other Oryzias species. While in situ hybridization showed THEA2 is expressed ubiquitously in medaka embryos, RT-PCR indicated greater THEA2 expression in the brown tissue homologous to mammalian BAT than in the other tissues in adult medaka (data not shown). In the structural predictions for the THEA2, we found that the two SNPs indicated for the human and medaka proteins are located at the junction between the Acyl-CoA hydrolase structural domains in a loop predicted to be highly flexible. There, a G-D (in humans) or L-P change (in medaka) is likely to affect the dynamics of the protein chain and influence (1) the interaction between domains and/or (2) the transmission of conformational changes. We speculate that the amino acid change that affects protein flexibility may be related to temperature adaptation.

For another gene, RTTN, we found even more remarkable regional differentiation. The phylogenetic network adding nine individuals from the northern Japanese population and one southern Japanese population indicates the nucleotide changes in the RTTN gene among geographical populations; each population forms a separate cluster and is separated by unique amino acid changes (Fig. 3). According to bioinformatic predictions, the RTTN protein is comprised of armadillo-like repeats separated in a few places by disordered loops (Fig. 4). A78 is partially buried and its substitution may destabilize the protein structure. S92 is located on the surface and is predicted to be phosphorylated; hence, its substitution may affect structure

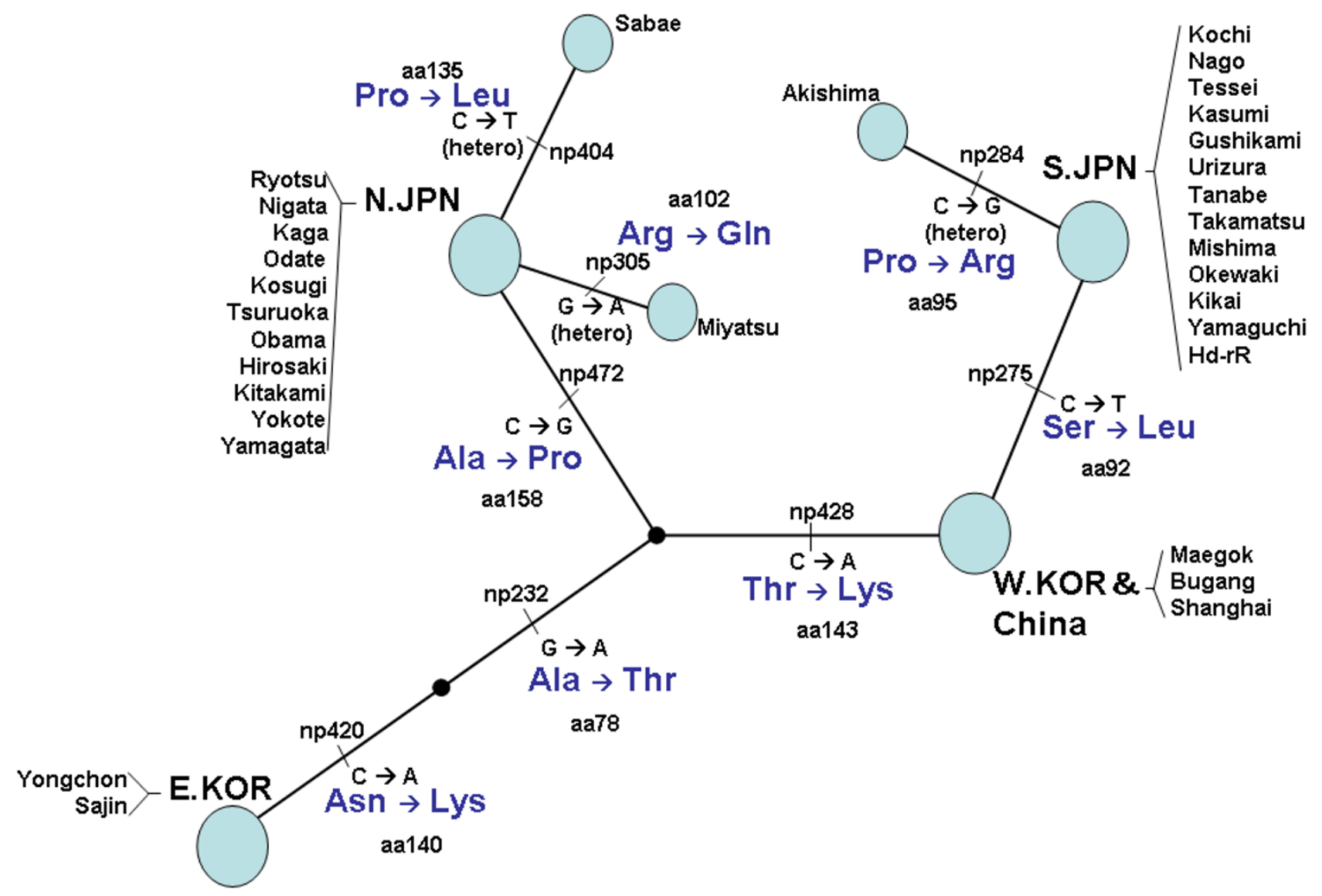

\section{Figure 3}

Phylogenetic network of RTTN based on nucleotide sequences from exons $3+4$ (27 I bp). The circle represents geographical regional strains (N.JPN: northern Japanese population; S.JPN: southern Japanese population; W.KOR: western Korean; E.KOR: eastern Korean). "np" represents the nucleotide position number. The "aa" numbers are the amino acid sequence positions. 


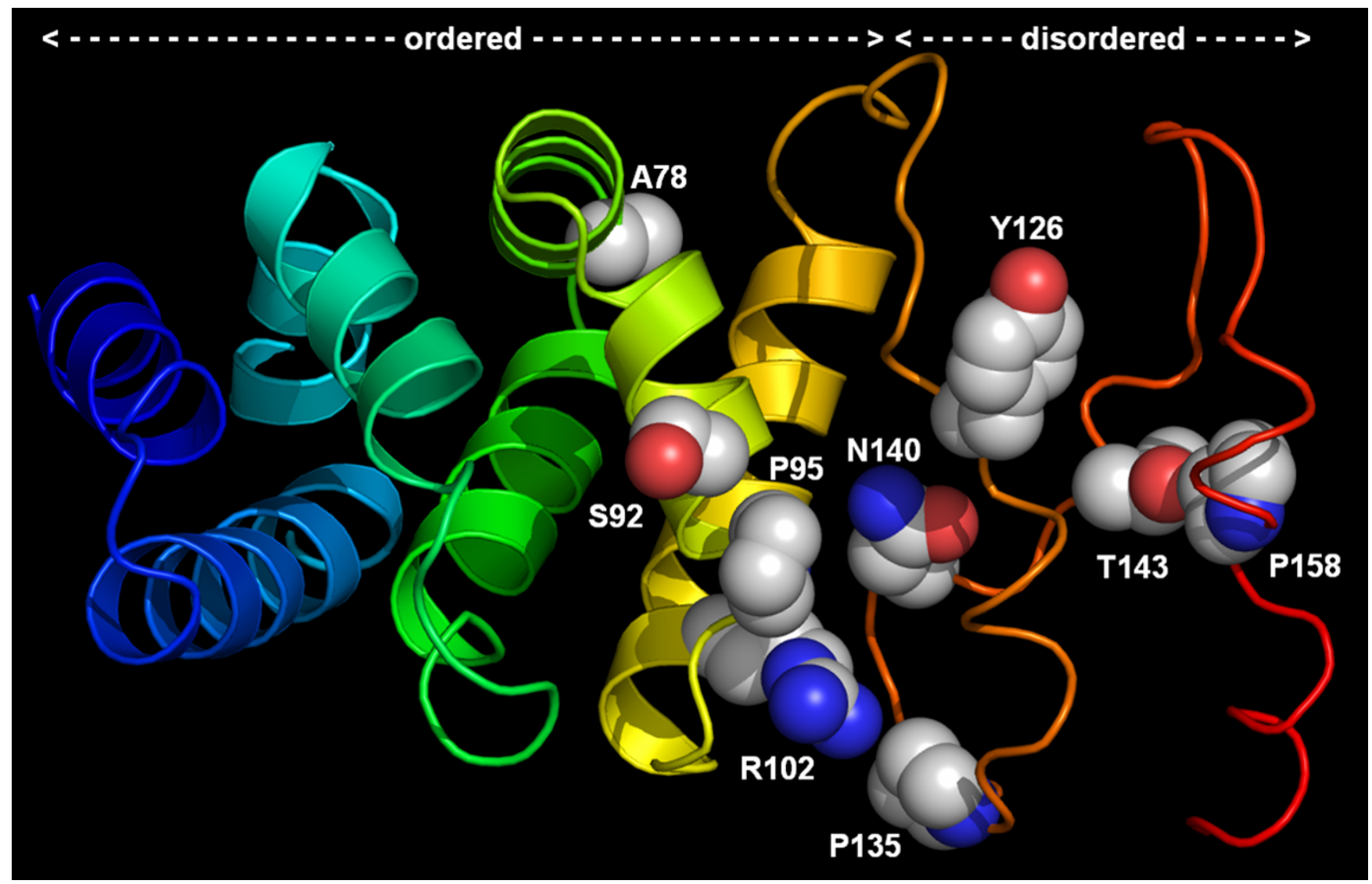

Figure 4

Structure prediction for RTTN: a well-folded globular part (armadillo-like repeats, aa I - I20) and an unstructured linker (aa I2I - I66). The protein chain is colored from blue ( $N$-terminus) to red (C-terminus). $\alpha$-helices are shown as ribbons. Side chains of residues substituted because of SNPs are shown in the "spacefill" representation and labeled; C, O, and $\mathrm{N}$ atoms are shown in gray, red, and blue, respectively. The positions of amino acid changes and the medaka populations sharing these changes are as follows: aa78, Thr: E.KOR, Ala: Others; aa92, Ser: S.JPN, Leu: Others; aa95, Arg: Akishima, Pro: Others; aa 102, GIn: Miyatsu, Arg: Others, aa I35, Leu: Sabae, Pro: Others; aa I40, Lys: E.KOR, Asn: Others; aa I43, Thr: E.KOR, N.JPN, Lys: W.KOR \& China, and S.JPN; aa I58, Ala: N.JPN, Pro: Others.

and/or function by removing a site of posttranslational modification. N140, T143, and P158 are in the disordered loop. Substituting P158 with A may increase the flexibility of the main chain, the introduction of K140 and K143 may increase the entropy of the side chain, and substitution of T143 (predicted to be phosphorylated) may remove a site of posttranslational modification. Thus, substitutions of all these residues are predicted to influence the dynamics of the loop and thus its ability to bind to other molecules or to respond to changes in the environment.

To gain further insight into whether natural selection is involved in the observed nucleotide variations, we plotted the average number of nonsynonymous nucleotide differences per number of nonsynonymous sites $\left(d_{N}\right)$ against the average number of synonymous nucleotide differences per number of synonymous sites $\left(d_{S}\right)$ estimated for the 11 genes among the 27 medaka strains (Fig. 5). Seven of the 11 genes including THEA2 showed an average $d_{\mathrm{N}} / d_{\mathrm{S}}$ of less than 1, suggesting that the seven genes are under purifying selection. In RTTN, in contrast, there are only nonsynonymous differences in the genomic regions examined (exons 3 and 4: 271 bp in total); in more than half of the population pairs, the $d_{\mathrm{N}} / d_{\mathrm{S}}$ ratios are significantly greater than 1 (Z-test; $p<0.05$ ). The $d_{\mathrm{N}} / d_{\mathrm{S}}$ ratios of the LTC and the GRK4 genes are also greater than 1, but these are not statistically significant at 5\% level for any pair. We have sequenced the entire RTTN cDNA for seven individual medaka from five geographical populations. Although there are synonymous variations in the other exons, the $d_{\mathrm{N}} / d_{\mathrm{S}}$ ratios are overall greater than 1 , and in 


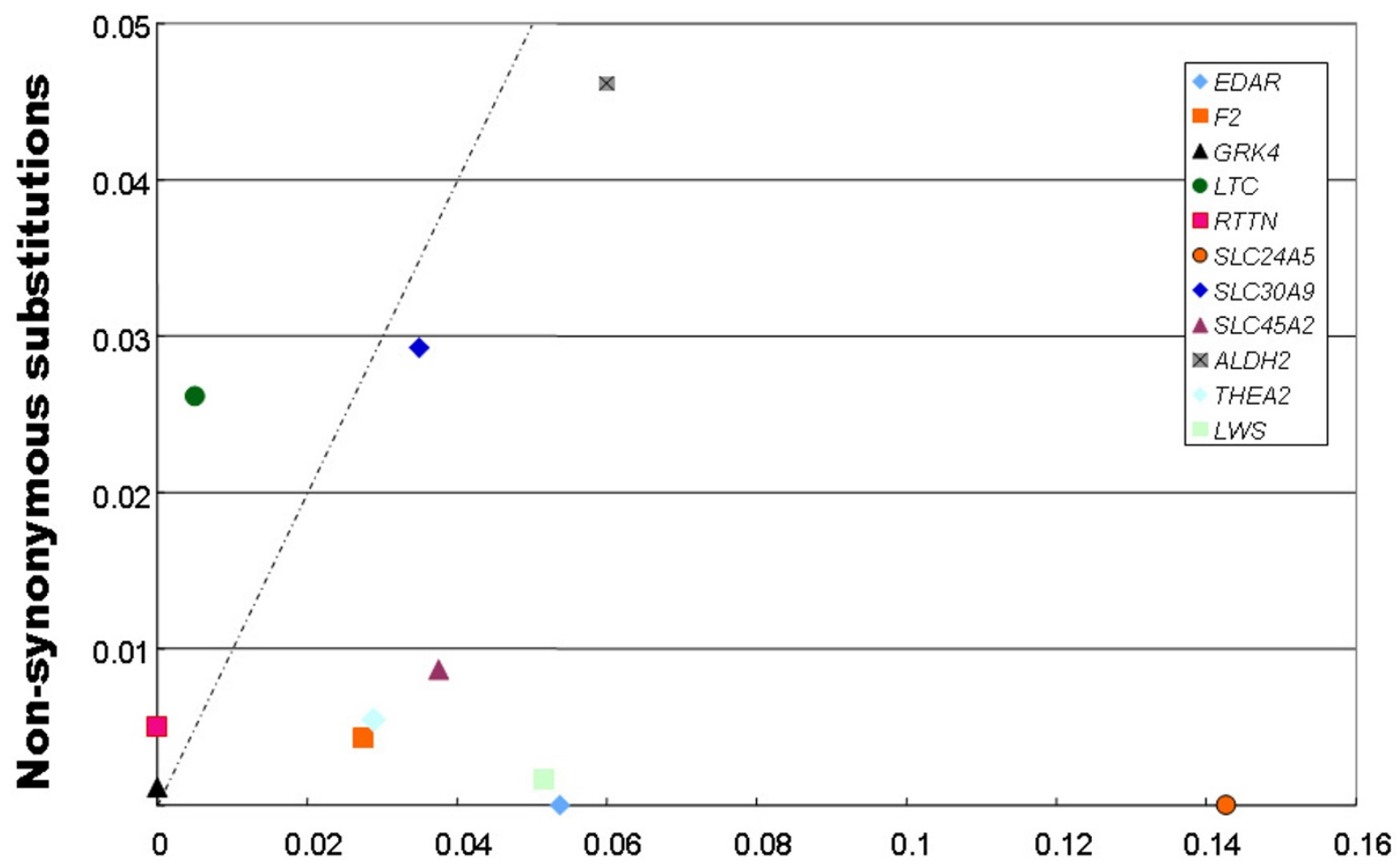

Synonymous substitutions

Figure 5

Synonymous ( $\mathrm{X}$ axis) and nonsynonymous ( $\mathrm{Y}$ axis) substitution ratios estimated by the Nei - Gojobori method. A dN/dS ratio significantly greater than I is a convincing indicator of positive selection.

Table 2: The dN - dS values (upper diagonal) and the significance (lower diagonal) based on RTTN cDNA (5.8 kb) sequences

\begin{tabular}{|c|c|c|c|c|c|c|c|c|}
\hline & Population samples & Nigata & Iwaki & Mishima & Nago & Shanghai & Maegok & Yongchon \\
\hline \multirow[t]{2}{*}{ N.JPN } & Nigata & & 1.622 & 1.712 & 1.685 & 1.116 & 1.835 & 1.87 \\
\hline & Iwaki & 0.054 & & 2.988 & 1.674 & 0.847 & 1.567 & 1.077 \\
\hline \multirow[t]{2}{*}{ S.JPN } & Mishima & 0.045 & $0.002 *$ & & 2.005 & 0.91 & 1.507 & 1.072 \\
\hline & Nago & $0.047^{*}$ & $0.048 *$ & $0.024^{*}$ & & 1.009 & 1.886 & 0.876 \\
\hline China & Shanghai & 0.133 & 0.199 & 0.182 & 0.157 & & 0.549 & 1.218 \\
\hline W.KOR & Maegok & $0.035^{*}$ & 0.06 & 0.067 & $0.031 *$ & 0.292 & & 2.103 \\
\hline E.KOR & Yongchon & $0.032 *$ & 0.142 & 0.143 & 0.191 & 0.113 & $0.019 *$ & \\
\hline
\end{tabular}

* represents $5 \%$ level significance of $p$-value in Z-test. "N. JPN" and "S.JPN" represent North and South Japan medaka, respectively. "W.KOR" and "E.KOR" represent West and East Korea medaka, respectively 
nine of the 21 pairs they are statistically significant $(p<$ 0.05 ; Table 2). These results suggest that RTTN is under positive selection in medaka.

Although its exact function is not known, RTTN is reported to be involved in determining the rotation of the body axis and the left-right asymmetry of internal organs during the embryonic development of mice [19]. The conspicuous differentiation of RTTN alleles among human populations also suggests differential natural selection acting on different populations: at a nonsynonymous SNP site (rs3911730) in the RTTN exon 3, the A/A genotype occurs in $90 \%$ of Africans, $2 \%$ of Europeans and is absent in Asians, while the $\mathrm{C} / \mathrm{C}$ genotype occurs in 3\% of Africans, $80 \%$ of Europeans and $100 \%$ of Asians.

Previous studies have reported that genes identified in fish through "forward genetic" analysis of phenotypic mutants are involved in forming variations of related phenotypes in humans, e.g. of skin pigmentation [20-24] and epithelial development [25]. Our approach in this study is an extension of these previous studies, as a form of "reverse genetics" of genes that show, as a signature of natural selection acting on them, a prominent level of diversification in the allele frequency among populations with different ecological histories in both fish and humans. We found that out of 11 genes in our analysis, the medaka THEA2 gene has a nonsynonymous polymorphic site at exactly the same position as its ortholog in humans, and the RTTN gene shows signs of population differentiation that can be explained plausibly by natural selection. The aim of our analysis is not to demonstrate evidence of natural selection in medaka, but to indicate that medaka is a marvelous resource as a "natural library" of genetic diversity, and this approach is efficient enough to find candidate genes targeted by natural selection in both humans and medaka. The exact function of the genes and the exact nature of the functional differences between alleles can be studied more feasibly in medaka, where crossing experiments between different genotypes of interest and transgenic techniques have already been established $[7,8]$. This method can be applied to any polymorphic gene in humans, and larger-scale and more systematic screening of orthologous gene polymorphisms in medaka will find various target genes for further functional analyses. As the medaka has been widely used for carcinogenesis and ecotoxicological studies [7], for example, in screening for genetic variants concerning medaka carcinogenesis and ecotoxins, it could also be used for testing variations in drug response in humans. Thus, we conclude that the medaka is a good vertebrate model of the functional diversity caused by human DNA polymorphisms that have been identified by recent resequencing and typing efforts.

\section{Authors' contributions}

HO conceived, and SK and HM formed the project. SO and HM provided the medaka resources. HO, YM, and HM designed the experiments. KW and YM performed PCRs and sequencing. For THEA2 and RTTN, YM performed the RT-PCRs and cDNA sequencing, YA and HN performed WISH. JMB performed protein structure predictions. HO and YM analyzed the data and wrote the paper. All authors read and approved the final manuscript.

\section{Additional material}

\section{Additional file 1}

Sampling map of regional strains for Oryzias latipes. Four strains (Nigata, Ryotsu, Kaga and Odate) are from the Northern Japanese population, and 15 strains (Tanabe, Takamatsu, Tessei, Kasumi, Uridura, Iwaki, Mishima, Hagi, Okewaki, Kikai, Nago, Kochi, Yamaguchi, Akishima and Gushikami) are from the Southern Japanese population. Two strains (Yongchon and Sajin) are from the Eastern Korean population, and three strains (Maegok, Bugang and Shanghai) are from Western Korean and Chinese populations. For the RTTN gene, we examined nine additional individuals from seven wild strains (Kosugi, Tsuruoka, Obama, Hirosaki, Kamikita, Yokote, Yamagata) from the Northern Japanese population.

Click here for file

[http://www.biomedcentral.com/content/supplementary/17560500-2-88-S1.tiff]

\section{Additional file 2}

Sampling map of regional strains for closely related species. Click here for file

[http://www.biomedcentral.com/content/supplementary/17560500-2-88-S2.tiff]

\section{Acknowledgements}

This work was supported by a Grant-in-Aid for Scientific Research (A) from the Japan Society for the Promotion of Science (JSPS) (192070 I8) to SK, by a Grant-in-Aid for Scientific Research (C) from JSPS (19570226) to HO, and by a Grant-in-Aid for Scientific Research in the Priority Area "Comparative Genomics" (\#0I5) from the Ministry of Education, Culture, Sports, Science and Technology of Japan (MEXT) to HM. We thank Professor Emeritus Akihiro Shima and Dr. Atsuko Shimada (the University of Tokyo) for their efforts on keeping medaka stocks from wild populations.

\section{References}

I. The_international_HapMap_consortium: A haplotype map of the human genome. Nature 2005, 437:1299-1320.

2. Frazer KA, Ballinger DG, Cox DR, Hinds DA, Stuve LL, Gibbs RA, Belmont JW, Boudreau A, Hardenbol P, Leal SM, et al.: A second generation human haplotype map of over 3.I million SNPs. Nature 2007, 449:85I-86I.

3. Conrad DF, Jakobsson M, Coop G, Wen X, Wall JD, Rosenberg NA, Pritchard JK: A worldwide survey of haplotype variation and linkage disequilibrium in the human genome. Nat Genet 2006, 38: $1251-1260$.

4. McVean G, Spencer CC, Chaix R: Perspectives on human genetic variation from the HapMap Project. PLoS Genet 2005, I:e54.

5. Voight BF, Kudaravalli S, Wen X, Pritchard JK: A map of recent positive selection in the human genome. PLoS Biol 2006, 4:e72. 
6. Williamson SH, Hubisz MJ, Clark AG, Payseur BA, Bustamante CD, Nielsen R: Localizing recent adaptive evolution in the human genome. PLoS Genet 2007, 3:e90.

7. Wittbrodt J, Shima A, Schartl M: Medaka - a model organism from the far East. Nat Rev Genet 2002, 3:53-64.

8. Shima A, Mitani $H$ : Medaka as a research organism: past, present and future. Mech Dev 2004, I 2 I:599-604.

9. Naruse K, Hori H, Shimizu N, Kohara Y, Takeda H: Medaka genomics: a bridge between mutant phenotype and gene function. Mech Dev 2004, 1 21:619-628.

10. Matsumoto $\mathrm{Y}$, Fukamachi S, Mitani H, Kawamura S: Functional characterization of visual opsin repertoire in Medaka (Oryzias latipes). Gene 2006, 371:268-278.

11. Kasahara M, Naruse K, Sasaki S, Nakatani Y, Qu W, Ahsan B, Yamada T, Nagayasu Y, Doi K, Kasai Y, et al.: The medaka draft genome and insights into vertebrate genome evolution. Nature 2007, 447:7|4-7|9.

12. Thompson JD, Higgins DG, Gibson TJ: CLUSTAL W: improving the sensitivity of progressive multiple sequence alignment through sequence weighting, position-specific gap penalties and weight matrix choice. Nucleic Acids Res 1994, 22:4673-4680.

13. Nei M, Gojobori T: Simple methods for estimating the numbers of synonymous and nonsynonymous nucleotide substitutions. Mol Biol Evol I 986, 3:4I8-426.

14. Tamura K, Dudley J, Nei M, Kumar S: MEGA4: Molecular Evolutionary Genetics Analysis (MEGA) software version 4.0. Mol Biol Evol 2007, 24:1596-1599.

15. Kurowski MA, Bujnicki JM: GeneSilico protein structure prediction meta-server. Nucleic Acids Res 2003, 31:3305-3307.

16. Kosinski J, Cymerman IA, Feder M, Kurowski MA, Sasin JM, Bujnicki JM: A "FRankenstein's monster" approach to comparative modeling: merging the finest fragments of Fold-Recognition models and iterative model refinement aided by 3D structure evaluation. Proteins 2003, 53(Suppl 6):369-379.

17. Wallner $B$, Elofsson $A$ : Identification of correct regions in protein models using structural, alignment, and consensus information. Protein Sci 2006, I5:900-913.

18. Adams SH, Chui C, Schilbach SL, Yu XX, Goddard AD, Grimaldi JC, Lee J, Dowd P, Colman S, Lewin DA: BFIT, a unique acyl-CoA thioesterase induced in thermogenic brown adipose tissue: cloning, organization of the human gene and assessment of a potential link to obesity. Biochem J 200I, 360: I35-142.

19. Faisst AM, Alvarez-Bolado G, Treichel D, Gruss P: Rotatin is a novel gene required for axial rotation and left-right specification in mouse embryos. Mech Dev 2002, I I 3:15-28.

20. Fukamachi $S$, Shimada A, Shima A: Mutations in the gene encoding $B$, a novel transporter protein, reduce melanin content in medaka. Nat Genet 200I, 28:38I-385.

21. Lamason RL, Mohideen MA, Mest JR, Wong AC, Norton HL, Aros MC, Jurynec MJ, Mao X, Humphreville VR, Humbert JE, et al:: SLC24A5, a putative cation exchanger, affects pigmentation in zebrafish and humans. Science 2005, 310:1782-1786.

22. Nakayama K, Fukamachi $S$, Kimura $H$, Koda $Y$, Soemantri $A$, Ishida $T$ : Distinctive distribution of AIMI polymorphism among major human populations with different skin color. J Hum Genet 2002, 47:92-94.

23. Fukamachi $S$, Kinoshita M, Tsujimura $T$, Shimada A, Oda S, Shima A, Meyer A, Kawamura S, Mitani H: Rescue From Oculocutaneous Albinism Type 4 Using Medaka slc45a2 cDNA Driven by Its Own Promoter. Genetics 2008, 178:76I-769.

24. Miller CT, Beleza S, Pollen AA, Schluter D, Kittles RA, Shriver MD, Kingsley DM: cis-Regulatory changes in Kit ligand expression and parallel evolution of pigmentation in sticklebacks and humans. Cell 2007, I3 I: | I79-। I89.

25. Kondo S, Kuwahara $Y$, Kondo M, Naruse K, Mitani H, Wakamatsu $Y$, Ozato K, Asakawa S, Shimizu N, Shima A: The medaka rs-3 locus required for scale development encodes ectodysplasin-A receptor. Curr Biol 200 I, I I: I202-1206.

26. Wright S: Evolution in Mendelian Populations. Genetics 1931, 16:97-159.

27. Sabeti PC, Schaffner SF, Fry B, Lohmueller J, Varilly P, Shamovsky O, Palma A, Mikkelsen TS, Altshuler D, Lander ES: Positive natural selection in the human lineage. Science 2006, 3 I 2:1614-1620.

28. Han Y, Gu S, Oota H, Osier MV, Pakstis AJ, Speed WC, Kidd JR, Kidd KK: Evidence of positive selection on a class I ADH locus. Am J Hum Genet 2007, 80:44I-456.
29. Oota H, Pakstis AJ, Bonne-Tamir B, Goldman D, Grigorenko E, Kajuna SL, Karoma NJ, Kungulilo S, Lu RB, Odunsi K, et al.: The evolution and population genetics of the ALDH2 locus: random genetic drift, selection, and low levels of recombination. Ann Hum Genet 2004, 68:93-109.

30. Myles S, Tang K, Somel M, Green RE, Kelso J, Stoneking M: Identification and analysis of genomic regions with large betweenpopulation differentiation in humans. Ann Hum Genet 2008, 72:99-110.

Publish with Bio Med Central and every scientist can read your work free of charge

"BioMed Central will be the most significant development for disseminating the results of biomedical research in our lifetime. "

Sir Paul Nurse, Cancer Research UK

Your research papers will be:

- available free of charge to the entire biomedical community

- peer reviewed and published immediately upon acceptance

- cited in PubMed and archived on PubMed Central

- yours - you keep the copyright 